\title{
Diacronie
}

Studi di Storia Contemporanea

$N^{\circ} 8,4 \mid 2011$

Le sembianze di Clio: approcci alla storia

\section{Marco Adorni, Voci di vetro. Testimonianze di vita alla Bormioli Rocco di Parma}

Jacopo Bassi

\section{(2) OpenEdition}

\section{Edizione digitale}

URL: http://journals.openedition.org/diacronie/3487

DOI: $10.4000 /$ diacronie.3487

ISSN: 2038-0925

\section{Editore}

Association culturelle Diacronie

\section{Notizia bibliografica digitale}

Jacopo Bassi, « Marco Adorni, Voci di vetro. Testimonianze di vita alla Bormioli Rocco di Parma », Diacronie [Online], № 8, 4 | 2011, documento 9, Messo online il 29 octobre 2011, consultato il 23 septembre 2020. URL : http://journals.openedition.org/diacronie/3487 ; DOI : https://doi.org/10.4000/ diacronie.3487 


\section{Diacronie}

N. 8 | 4|2011 Le sembianze di Clio: approcci alla storia

9/

\section{RECENSIONE:}

\section{Marco ADORNI, Voci di vetro. Testimonianze di vita alla Bormioli Rocco di Parma, Milano, Punto Rosso, 2010, 223 pp.}

a cura di Jacopo BASSI *

Quando hanno chiuso la Bormioli mi son venuti $i$ brividi. Secondo me questo è avvenuto perché c'era una storia dietro, la "Luigi" non l'aveva, la Barilla nemmeno, le altre aziende non l'avevano. La Bormioli è nata lì nel Novecento. Lì c'è stata una continuità storica, ogni famiglia di Parma ha avuto qualcuno che ci ha lavorato. La Bormioli era la fabbrica di Parma. ${ }^{1}$

Testimonianza di Giancarlo Meruni

Voci di vetro raccoglie le testimonianze dell'esperienza di lavoro di alcuni ex dipendenti della vetreria Bormioli Rocco di Parma; curatore del volume è Marco Adorni, ricercatore del Centro Studi Movimenti di Parma e collaboratore della facoltà di Scienze della formazione dell'Università di Bologna. Il volume, tuttavia, non è una semplice raccolta di documentazione orale affiancata dalle macrovicende storiche della Bormioli: alla base del volume c'è la volontà di decostruire la storia della vetreria in una pluralità di storie diverse, quelle narrate dagli intervistati, ex dipendenti della fabbrica.

Il materiale raccolto non era stato originariamente concepito per questa pubblicazione, bensì per la realizzazione del documentario la vetreria ${ }^{2}$; la finalità cronologica era quella di coprire tutto l'arco cronologico di esistenza dell'azienda nel

\footnotetext{
${ }^{1}$ ADORNI, Marco, Voci di vetro. Testimonianze di vita alla Bormioli Rocco di Parma, Milano, Punto Rosso, 2010, p. 165.

${ }^{2}$ La vetreria, di Marco Adorni, William Gambetta, Mario Ponzi, Italia, 2008
} 
XX secolo, dalla fase di lavorazione manuale del vetro, negli anni Trenta, sino alla chiusura, avvenuta nel 2004.

L'intenzione alla base del progetto, esplicitata da Adorni con un chiaro riferimento a Benjamin, è quella di non «fornire interpretazioni o commenti al lettore, ma limitarsi a selezionare, arrangiare, montare e far vedere le immagini scelte»3. L'approccio proposto è quindi filologicamente attento tanto al rispetto delle parole e delle linee narrative scelte dagli intervistati, quanto - in separata sede - al commento e alla contestualizzazione della testimonianza. L'obiettivo era dunque quello di realizzare un'edizione critica delle testimonianze orali di quindici ex dipendenti della vetreria Bormioli Rocco.

La metodologia adottata nelle interviste si è basata sulla formulazione di interrogazioni asistematiche, variabili in ragione della traiettoria assunta dal dialogo. La fedeltà della trasposizione delle testimonianze è stata mantenuta anche attraverso la conservazione del dialetto parmigiano, trascritto fedelmente e di cui viene fornita una puntuale traduzione in nota. Il mantenimento dei passaggi del dialogo in dialetto, unitamente alla descrizione dei gesti e degli atteggiamenti non verbali, permette di cogliere alcune coloriture del discorso diversamente non comprensibili con il solo ausilio della parola scritta. A corredo del volume è inserito un apparato iconografico, con fotografie provenienti dall'Archivio Biblioteca Bormioli Rocco e dagli archivi privati degli ex dipendenti.

Il necessario intervento critico rispetto alla soggetività delle narrazioni è stato operato ex post e non nel corso della realizzazione dell'intervista: quest'operazione, infatti, non ambiva a una sconfessione della memoria dell'intervistato, ma, al contrario, alla «comprensione della soggettività di chi racconta storie»4.

Il lavoro si sofferma sulla costruzione dei sentimenti collettivi di appartenenza all'azienda, su come questi abbiano originato una vera e propria visione del mondo legata alla Bormioli e su come gli eventi di conflittualità sociale abbiano creato delle discontinuità rispetto alla visione unificante della vita di fabbrica.

L'eterogeneità dei testimoni - diversi per classi sociali, ruolo svolto in fabbrica e periodo di impiego alla Bormioli Rocco- ha richiesto un ulteriore sforzo di contestualizzazione, che si è aggiunto alle difficoltà derivanti dalle differenze culturali tra l'intervistatore e gli intervistati.

Ogni testimonianza è corredata da una breve introduzione - che chiarisce il contesto in cui è stata realizzata l'intervista, lo stato d'animo dell'intervistato, le

\footnotetext{
3 Ibidem, p. 12.

4 Ibidem, p. 13. 
persone presenti durante la registrazione e il luogo in cui è stata realizzata - e da una postfazione, curata dall'autore, utile alla contestualizzazione e all'interpretazione di quanto emerso nell'intervista.

Le testimonianze sono raccolte in due capitoli tematici, Comunità e Conflitti, contenitori ideali, puramente funzionali a contrassegnare l'esperienza lavorativa a seconda della rappresentazione fornita dagli ex dipendenti della propria "vita di fabbrica" alla Bormioli Rocco. Marco Adorni fornisce, nella Premessa, una spiegazione dei criteri alla base dell'inserimento nell'uno o nell'altro gruppo; questo risulta utile soprattutto per alcuni casi di testimonianze caratterizzate da una prospettiva multiforme.

Il sito industriale della Bormioli Rocco - dinastia di vetrai parmigiani con più di sei secoli di storia alle spalle - sorse nel quartiere San Leonardo, caratterizzandolo e conferendogli i connotati popolari e operai che lo avrebbero contraddistinto per buona parte del XX secolo, come ricorda Giancarlo Merlini: «la Bormioli era la fabbrica di Parma. Il San Leonardo era il cuore. Quando io sono arrivato, nel '67, la Bormioli era in campagna, pensate cos'è avvenuto. E molta di questa gente è venuta da fuori, campagna e montagna, e ha comprato casa lì. La gente che lavorava vedeva cosa facevano le famiglie in casa. Il quartiere è sempre stato solidale nei momenti di lotta, anche il prete. Era gente che lavorava dentro ma viveva fuori»5. Alla base del progetto di Rocco Bormioli vi era infatti l'idea di città-fabbrica, costruita intorno a regole di comportamento e sociali che modellarono l'inurbamento dei contadini assunti in Bormioli: « [...] non a caso, nonostante il progetto produttivo della Bormioli e quello del settore agro-alimentare avessero ben pochi punti in comune, la direzione aziendale preferiva il bracciante al disoccupato dei borghi popolari dell'Oltretorrente cittadino, storicamente ribelle e poco propenso all'accettazione passiva del comando autoritario ${ }^{6}$. Fu a partire da questo contesto che si consolidò l'identità comunitaria dei bormiolini, intorno alla quale si sviluppano le testimonianze raccolte nel primo macrocapitolo, Comunità.

È proprio il sentimento identitario del noi-bormiolini - secondo Adorni estintosi solo con il decentramento produttivo iniziato al termine degli anni Settanta - a creare i presupposti per una costruzione identitaria in grado di andare oltre i sentimenti di appartenenza di classe. Spesso l'elemento accomunante risulta essere quello della condivisione della durezza del lavoro: «la vetreria è un ambiente infernale. Il primo impatto che uno ha è il rumore. Si parla ma non si capisce. Solo muovendo la bocca si

5 Ibidem, p. 165.

${ }^{6}$ Ibidem, p. 26. 
riusciva a capire quello che l'altro faceva. E poi l'altro elemento è il caldo. Temperature altissime e spesso l'intervento sulla macchina si faceva con la macchina in movimento»7. Le testimonianze permettono tuttavia di cogliere un'oscillazione, nei ricordi, tra l'evocazione della "fabbrica della morte" e una forma di nostalgia per il lavoro in vetreria e per i legami d'amicizia e solidarietà creatisi in fabbrica. Giorgio Giliotti parlando del clima all'interno dell'azienda, si esprime in questi termini:«la Bormioli è come un paese. È come la caserma. È vero che ci sono quelli che, non adeguandosi alla compagnia [...] puoi anche restare insoddisfatto, ma fondamentalmente, era una famiglia perché se c'era un guaio non eri mai solo. C'era molta solidarietà» ${ }^{8}$.

La conflittualità interna alla Bormioli Rocco si è espressa fra differenti organi, ma anche all'interno di questi stessi: di qui la scelta di Marco Adorni di declinare al plurale, Conflitti, la macrosezione dedicata agli scontri e alla dialettica interna alla fabbrica. Il 1949, con l'occupazione operaia della vetreria e il successivo allontanamento delle guide del movimento operaio, è certamente il punto di partenza delle proteste e delle rivendicazioni del dopoguerra; le testimonianze permettono però di ripercorrere sempre attraverso le soggettività degli intervistati - anche le vicende degli anni Sessanta e Settanta, caratterizzate dalle lotte più dure e dall'avvento dell'automazione in fabbrica, e gli anni Ottanta e Novanta, quelli della ristrutturazione e del decentramento. Proprio riguardo all'occupazione della fabbrica nel 1949 le interviste di Adorni ci restituiscono una poliedrica ricostruzione delle vicende di quei giorni: protagonisti, piccoli e grandi eventi e dettagli di quei giorni mutano da testimonianza a testimonianza, restituendo una sorta di epica collettiva dell'occupazione.

Il maggior merito del volume è di restituire, attraverso la plurale e multisfaccettata Weltanschauung dei lavoratori della Bormioli Rocco, le storie - e non la storia dell'epopea industriale della vetreria parmigiana.

\footnotetext{
7 Ibidem, p. 156.

8 Ibidem, p. 131. 


\section{* L'autore}

Jacopo Bassi è dottore magistrale in Storia d'Europa. Si è laureato in Storia della Chiesa presso l’Università di Bologna nel 2008; attualmente collabora con la casa editrice Il Mulino.

URL: < http://www.studistorici.com/2009/02/24/jacopo_bassi >

\section{Per citare questo articolo:}

BASSI, Jacopo, «Recensione: Marco ADORNI, Voci di vetro. Testimonianze di vita alla Bormioli Rocco di Parma, Milano, Punto Rosso, 2010, 223 pp.», Diacronie. Studi di Storia Contemporanea: Le sembianze di Clio: approcci alla storia, 29/10/2011,

URL:<http://www.studistorici.com/2011/10/29/bassi_numero_8/ >

\section{Diacronie Studi di Storia Contemporanea $\mathcal{\beta}$ www.diacronie.it}

Risorsa digitale indipendente a carattere storiografico. Uscita trimestrale. redazione.diacronie@hotmail.it

Comitato di redazione: Marco Abram - Giampaolo Amodei - Jacopo Bassi - Luca Bufarale - Alessandro Cattunar - Alice De Rensis Barbara Galimberti - Deborah Paci - Fausto Pietrancosta - Martina Sanna - Matteo Tomasoni - Luca Zuccolo

Diritti: gli articoli di Diacronie. Studi di Storia Contemporanea sono pubblicati sotto licenza Creative Commons 2.5 .

(c) (1) $\odot$ Possono essere riprodotti a patto di non modificarne i contenuti e di non usarli per fini commerciali. La citazione di C. 